\title{
A new structural model for disorder in vaterite from first-principles calculations.
}

\author{
Raffaella Demichelis, ${ }^{* a}$ Paolo Raiteri, ${ }^{a}$ Julian D. Gale ${ }^{a}$ and Roberto Dovesi ${ }^{b}$ \\ Received (in $X X X, X X X)$ Xth $X X X X X X X X X 20 X X$, Accepted Xth $X X X X X X X X X 20 X X$ \\ DOI: 10.1039/b000000x
}

Both of the previously proposed $P b n m$ and $P 6_{5} 22$ ordered structures for vaterite are found to be unstable transition states using first-principles methods. Four stable structures are located, the lowest energy one being of $P 3_{2} 21$ symmetry. ${ }_{10}$ Since interconversion between these structures requires only thermal energy, this provides an additional source of disorder within the vaterite structure.

Calcium carbonate polymorphs (calcite, aragonite and vaterite) play an important role in the chemistry of the hydrosphere, 15 lithosphere and biosphere, as well as being a technological hindrance in the form of scale. Despite the rare geological occurrence of vaterite, it represents a relevant mineral in the formation of $\mathrm{CaCO}_{3}$ under biogenic conditions. For example, many living organisms are known to crystallise vaterite as a

20 component of their endo- or exo-skeleton, with biomolecules often acting as structural stabilisers. ${ }^{1}$ Moreover, the observed nucleation of vaterite from amorphous calcium carbonate (ACC) precursors makes it relevant to the understanding of non-classical nucleation and crystal growth mechanisms. ${ }^{2,3,4}$

25 Whereas the crystal structures of calcite and aragonite are ordered and well defined, that of vaterite remains a matter of debate, in spite of many experimental and theoretical studies carried out for more than half a century (see Table $\mathbf{1}$ for a concise summary). Generally, different interpretations of X-ray diffraction data 30 collected by various authors have suggested two candidate crystal systems; orthorhombic (Pbnm) and hexagonal $\left(\mathrm{Pb}_{3} / \mathrm{mmc}\right.$, $\mathrm{P}_{3} / m m c$ with partial occupancy of $\mathrm{CO}_{3}{ }^{2-}$ sites, or $\left.\mathrm{P}_{3} 22\right)$. On the basis of Raman spectroscopic studies, Behrens et al. ${ }^{5}$ concluded that the space group of vaterite can be neither $P 6_{3} / m m c$, nor ${ }_{35} \mathrm{P}_{3} 22$, whereas Gabrielli et al. ${ }^{6}$ agreed with Meyer, ${ }^{7}$ proposing a $\mathrm{P6}_{3} / \mathrm{mmc}$ structure with partial occupancy of $\mathrm{CO}_{3}{ }^{2-}$ sites, though with some residual ambiguities. Recent solid-state NMR data also supports the hexagonal structural model in preference to the orthorhombic one. ${ }^{8}$ On the contrary, Le Bail et al. ${ }^{9}$ propose that 40 the structure is indeed ordered and orthorhombic, but with three misaligned domains separated by $120^{\circ}$, thereby leading to an apparent hexagonal character. Based on this interpretation of their X-ray data, they proposed the Ama2 space group, while noting the presence of some unexplained superstructure reflections. ${ }_{45}$ Analysis of the vaterite Raman spectrum by Wehrmeister et al. ${ }^{10}$ shows that none of the current proposed structures are consistent with their data, which indicates the presence of three inequivalent
Table 1 Structures proposed for vaterite: space group $(S G)$ and lattice parameters $[\AA]$.

\begin{tabular}{|c|c|c|c|c|}
\hline Reference & $S G$ & $a$ & $b$ & $c$ \\
\hline Meyer $^{11}$ & Pbnm & 4.13 & 7.15 & 8.48 \\
\hline McConnell $^{12}$ & $\mathrm{P}_{3} 22$ & 7.135 & 7.135 & 8.524 \\
\hline Kahmi $^{13}$ Sato and Matsuda ${ }^{14}$ & $\mathrm{P}_{3} / m m c$ & 4.13 & 4.13 & 8.49 \\
\hline Bradley et al. ${ }^{15}$ & $\mathrm{P}_{3} 22$ & 7.135 & 7.135 & 8.524 \\
\hline Meyer, ${ }^{7}$ Gabrielli et al. ${ }^{6}$ & $\mathrm{P}_{3} / \mathrm{mmc}^{*}$ & 7.15 & 7.15 & 16.96 \\
\hline Dupont et al. ${ }^{16}$ & $\mathrm{PG}_{3} / \mathrm{mmc}^{*}$ & 7.169 & 7.169 & 16.98 \\
\hline Le Bail et al. ${ }^{9}$ & Ama2 & 8.7422 & 7.1576 & 4.1265 \\
\hline Medeiros et al. ${ }^{17}$ & $\mathrm{Pbnm}^{\dagger}$ & 4.531 & 6.640 & 8.480 \\
\hline Wang and Becker ${ }^{18}$ & $P 6_{5} 22^{\dagger}$ & 7.290 & 7.290 & 25.302 \\
\hline
\end{tabular}

${ }_{50}{ }^{*}$ Partial occupancy ${ }^{\dagger}$ First-principles calculation

carbonate groups.

In the last few years, Medeiros et al. ${ }^{17}$ and Wang and Becker ${ }^{18}$ have examined this problem by using first-principles calculations (pseudopotential plane-wave methodology, employing Density ${ }_{55}$ Functional Theory, DFT, at the level of the Generalized Gradient Approximation, GGA). In particular, the former study analysed the structural, electronic and optical properties of Pbnmstructured vaterite, whereas the latter investigators proposed the $\mathrm{P}_{5} 22$ space group as a result of an energetic analysis of different 60 hexagonal configurations. However, neither of these studies computed the vibrational spectra for the candidate structures. Not only can this be valuable for comparison to experimental data, but also turns out to be very revealing in terms of the stability of the proposed space groups, as will be demonstrated later.

${ }_{65}$ Here we re-examine all of the possible ordered structures of vaterite and thereby arrive at a new proposed model. The present calculations were performed with the quantum-mechanical $a b$ initio CRYSTAL09 program. ${ }^{19}$ All-electron Gaussian-type basis sets optimised for calcite ${ }^{20}$ were adopted, i.e. 86-511d(21)G for ${ }_{70} \mathrm{Ca}, 6-311 \mathrm{~d}(11) \mathrm{G}$ for $\mathrm{C}$ and $8-411 \mathrm{~d}(11) \mathrm{G}$ for O. Pbnm and $P 6_{5} 22-$ structured vaterite were computed first at the same level of theory as the previous first-principles calculations, ${ }^{17,18,21}$ obtaining very similar structural results. The structural and energetic analyses were then performed with the $\mathrm{PBEsol}^{22}$ functional, which is a 75 revised version of the widely used PBE functional specifically tailored for solids. This functional has been shown to provide accurate results for $\mathrm{H}$-free systems and, in general, correct relative stabilities. ${ }^{23,24}$ The corresponding hybrid functionals, $\mathrm{PBE}^{25}$ and PBEsol0, containing $25 \%$ of exact Hartree-Fock $80(\mathrm{HF})$ exchange, and B3LYP, ${ }^{26}$ were also used to validate the 
present results.

The DFT exchange-correlation contribution was evaluated by numerical integration over the unit cell volume, using a pruned grid with 75 radial (Gauss-Legendre radial quadrature) and 974 5 angular (Lebedev two-dimensional generation) points. Thresholds for the Coulomb and HF exchange series accuracy were set to $10^{-}$ ${ }^{8}, 10^{-8}, 10^{-8}, 10^{-8}$ and $10^{-16}$ for the exact, and to $10^{-18}$ for the bipolar-approximated, integral calculation. ${ }^{19}$ Reciprocal space was sampled according to a Monkhorst-Pack mesh with shrinking

10 factors of 8 and 4 for the orthorhombic and hexagonal (including subgroups) unit cells, respectively.

Structure optimisations were performed using analytical gradients with respect to atomic coordinates and unit-cell parameters, within a quasi-Newtonian scheme combined with Broyden${ }_{15}$ Fletcher-Goldfarb-Shanno ${ }^{27}$ Hessian updating. The default convergence criteria were used for both gradient components and nuclear displacements. Tightening these thresholds by an order of magnitude led to differences of the order of only $10^{-3} \AA$ in lattice parameters and $10^{-2} \mathrm{~kJ} / \mathrm{mol}$ in the electronic energy.

20 The harmonic vibrational frequencies were computed by diagonalising the dynamical matrix, obtained by central finite differencing of the analytic gradients with respect to atomic Cartesian coordinates. Scanning of geometries along imaginary normal modes was performed automatically by use of the ${ }_{25}$ SCANMODE option ${ }^{19}$ and the FINDSYM software was applied for symmetry analysis. ${ }^{28}$ Tolerances on the Self Consistent Field were set to $10^{-8}$ and $10^{-10}$ a.u. for optimisation and frequency calculation, respectively.

Table 2 Optimised structures of vaterite (PBEsol)

\begin{tabular}{cccccccc}
\hline & $P 6_{5} 22(T S)$ & $P 6_{5}$ & $P 3_{2} 21$ & $P b n m(T S)$ & $C 2 \mathrm{~cm}^{*}$ & $P 2_{1} 2_{1} 2_{1}$ & $P 112_{1}{ }^{\dagger}$ \\
$a$ & 7.1206 & 7.1121 & 7.1239 & 4.3907 & 4.5026 & 4.3668 & 7.1115 \\
$b$ & 7.1206 & 7.1121 & 7.1239 & 6.6091 & 6.3905 & 6.5831 & 7.1013 \\
$c$ & 25.4390 & 25.4089 & 25.3203 & 8.5029 & 8.4905 & 8.4282 & 25.3601 \\
Volume & 62.06 & 61.83 & 61.82 & 61.69 & 61.08 & 60.57 & 61.83 \\
Density & 2.674 & 2.684 & 2.684 & 2.691 & 2.71 & 2.740 & 2.684 \\
$\mathrm{Ca}-\mathrm{O}_{\min }$ & 2.304 & 2.284 & 2.280 & 2.346 & 2.334 & 2.344 & 2.269 \\
$\mathrm{Ca}-\mathrm{O}_{\max }$ & 2.423 & 2.687 & 2.622 & 2.367 & 2.422 & 2.603 & 2.625 \\
$\mathrm{Ca}-\mathrm{O}_{\mathrm{av}}$ & 2.359 & 2.384 & 2.392 & 2.358 & 2.375 & 2.407 & 2.385 \\
$\mathrm{CN}(\mathrm{Ca})$ & 6 & 6 & 6,7 & 6 & 6 & 7 & $6,7,8$ \\
$\mathrm{C}-\mathrm{O}_{\min }$ & 1.286 & 1.281 & 1.280 & 1.283 & 1.288 & 1.282 & 1.281 \\
$\mathrm{C}_{\mathrm{O}} \mathrm{O}_{\max }$ & 1.297 & 1.306 & 1.306 & 1.296 & 1.295 & 1.309 & 1.307 \\
$\mathrm{C}-\mathrm{O}_{\mathrm{av}}$ & 1.293 & 1.292 & 1.293 & 1.292 & 1.292 & 1.293 & 1.292
\end{tabular}

30 Lattice parameters $[\AA]$, volume per formula unit $\left[\AA^{3}\right]$, density $\left[\mathrm{g} \mathrm{cm}^{-3}\right]$, minimum, maximum and average bond distances $[\AA]$, Ca coordination number $(\mathrm{CN})$. Structural parameters obtained with the other functionals differ by less than $2 \%$. The experimental lattice parameters are given in Table 1. TS implies that a structure is a "transition state". *Ama2 is

35 permuted into $C 2 \mathrm{~cm}$ for ease of comparison to the other orthorhombic structures. ${ }^{\dagger} \gamma=60.34$ degrees

The calculated structures (PBEsol) are shown in Table 2 and their relative energies in Table 3. As shown by Wang and Becker, ${ }^{18}$ the $P 6_{5} 22$-structure for vaterite is more stable than the 40 Pbnm-form. However, while this previous work finds a stability difference of $71.7 \mathrm{~kJ} / \mathrm{mol}$, our results give values of the order of 2 $\mathrm{kJ} / \mathrm{mol}$ with all the functionals examined. Given that the experimental enthalpy differences between the extremes of amorphous calcium carbonate and calcite only span $\sim 15 \mathrm{~kJ} / \mathrm{mol}^{29}$ 45 we believe that the present energetics are more reasonable.

More significantly, both of the previously theoretically examined ordered structures for vaterite turn out to possess imaginary
Table 3 Energy differences $[\mathrm{kJ} / \mathrm{mol}]$ between structures relative to the unstable Pbmn configuration.

\begin{tabular}{cccccc}
\hline & PBE & PBEsol & PBE0 & PBEsol0 & B3LYP \\
$P 6_{5} 22(T S)$ & -2.05 & -2.04 & -2.36 & -2.31 & - \\
$P 6_{5}$ & -2.07 & -2.58 & - & -2.79 & - \\
$P 3_{2} 21$ & -2.94 & -3.50 & -3.34 & -3.63 & -2.68 \\
Pbnm $(T S)$ & 0.00 & 0.00 & 0.00 & 0.00 & 0.00 \\
Ama2 & +16.13 & +15.09 & - & +16.42 & - \\
s $4(T S)$ & -2.35 & -2.80 & - & -3.01 & - \\
$P 2_{1} 2_{1} 2_{1}$ & -0.97 & -2.27 & -1.22 & -2.11 & -0.11 \\
$P 112_{1}$ & -2.43 & -3.01 & - & -3.22 & -
\end{tabular}

50 TS implies that the structure is a "transition state".

phonons at the $\Gamma$ point and therefore are unstable transition states, rather than genuine minima. To be precise, four imaginary vibrational frequencies are found for the $P 6_{5} 22$ structure, in addition to the three translational modes, while the Pbnm ${ }_{55}$ configuration possesses one unstable mode. The hexagonal form exhibits imaginary modes at $63 i, 47 i, 47 i$ and $36 i \mathrm{~cm}^{-1}$ (PBEsol, with similar results for $\mathrm{PBE}$ ), while the unstable mode of the latter falls between $28 i$ and $50 i \mathrm{~cm}^{-1}$ (depending on the adopted functional). A small, real frequency $\left(17 \mathrm{~cm}^{-1}\right)$ is obtained as the 60 lowest vibrational mode for Pbnm-vaterite with the B3LYP functional, probably as a result of the larger predicted volume. Despite the marginal stability for this form, this structure remains the highest energy configuration with the B3LYP functional.

The above raises the question of why the instabilities were not ${ }_{65}$ previously observed in first-principles studies of vaterite? Aside from the fact that the phonon spectrum has not been previously computed, it might have been expected that the structures could naturally distort to lower symmetries due to the inherent numerical noise in the forces present in first-principles plane70 wave calculations. However, both prior studies employed codes that allow the space group symmetry to be constrained by default. Furthermore, the results of such calculations with regard to symmetry breaking have been shown to be sensitive to the details of the pseudopotential employed, whereas the present work 75 utilizes all-electron methods. ${ }^{30}$ To confirm our findings, we have computed the vibrational frequencies for the $P 6_{5} 22$ structure of Wang and $B e c k e{ }^{18}$ using the same computational approach as given in their paper. This also yields several imaginary modes demonstrating that the structure is only stable due to the space 80 group being automatically constrained.

Having determined that both of the widely considered ordered structures for vaterite are unstable, the next step is to determine the energy minima that are connected via these transition states. First, we performed a scan along the imaginary mode of the ${ }_{85}$ Pbnm form, which is symmetry forbidden by the inversion centre. By reducing the point symmetry from $D_{2 h}$ to $D_{2}$, the minimum energy structure was located and determined to belong to the space group $P 2_{1} 2_{1} 2_{1}$, and is more stable than $P b n m$-vaterite by between 0.1 and $2.3 \mathrm{~kJ} / \mathrm{mol}$, depending on the adopted functional. 90 As shown in Table 2, the two orthorhombic structures are very similar, with the $P 2_{1} 2_{1} 2_{l}$ one being denser and allowing $\mathrm{Ca}$ and $\mathrm{C}$ to assume a more distorted configuration, as shown by the bond length distribution and coordination number. The alternative orthorhombic model (Ama2), recently proposed by Le Bail et al., 95 is indeed a distinct stable energy minimum. However, it is found to be $\sim 16 \mathrm{~kJ} / \mathrm{mol}$ less stable than Pbnm-vaterite, and so can be discounted as a viable structure for this material. 

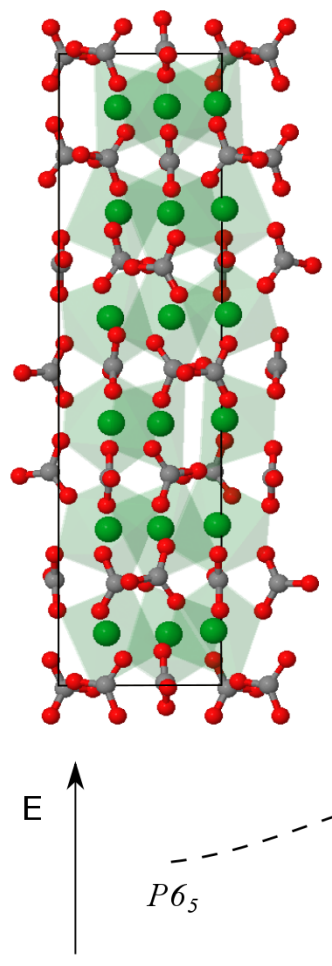



$\mathrm{PG}_{5} 22$

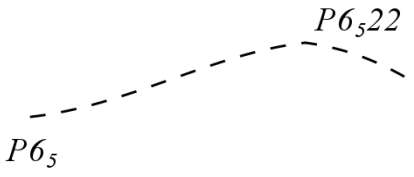

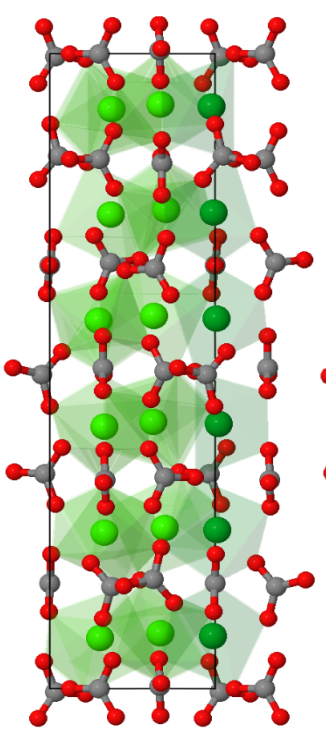
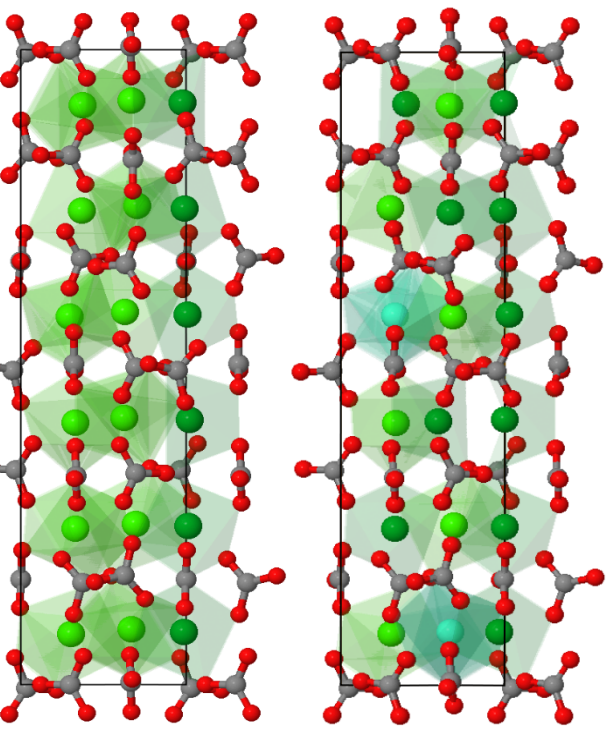

$\checkmark$

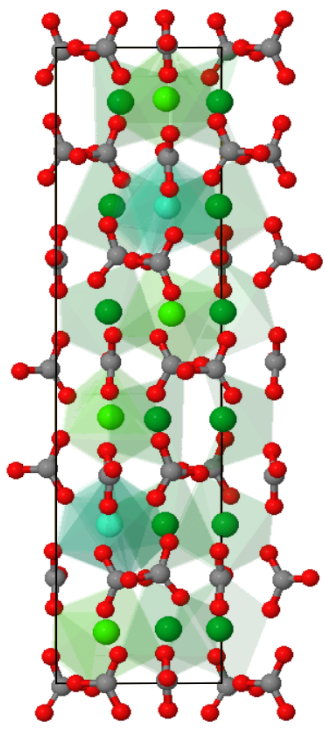

Ca coordination

6

7

8

$\bar{P} \overline{1} 12_{1}$

Figure 1 Graphical representation of the hexagonal vaterite structures. A view of the $18 \mathrm{Ca}$ atoms in the unit cell, with the corresponding coordination polyhedra, projected on to the $a c$ plane. 6-, 7-, and 8-fold coordinated $\mathrm{Ca}$ atoms and polyhedra are shown in different shades of green; $\mathrm{C}$ and $\mathrm{O}$ atoms are grey and red, respectively.

For the case of the hexagonal $\mathrm{P6}_{5} 22$-structure the situation is more complicated as there are four different imaginary modes leading to distinct minima. As a result of the scans along these four modes (all corresponding to symmetry-forbidden rotations of $\mathrm{CO}_{3}{ }^{2-}$ groups), three minimum energy structures, with space groups of $P 112_{1}, P 6_{5}$ and $P 3_{2} 21$, were obtained. A fourth structure (labelled in the 5 following discussion as $s 4$ ) turned out to correspond to a $2^{\text {nd }}$-order saddle point and so was not further analysed in detail.

$P 3_{2} 21$-vaterite, shown in Figure 1, is found to be the lowest energy structure (the unit cells and coordinates of all of the minima are reported in the Supplementary Information). Differences between $\mathrm{P6}_{5} 22$-vaterite and the structures belonging to its subgroups are quite small, mainly corresponding to more distorted positions of $\mathrm{O}$ atoms, whereas $\mathrm{Ca}$ and $\mathrm{C}$ atoms largely remain on the equivalent higher symmetry sites. This is shown by the varying coordination of $\mathrm{Ca}$ atoms and the bond length distribution.

10 According to the $\mathrm{Ca}-\mathrm{O}$ distances in the other $\mathrm{CaCO}_{3}$ polymorphs, we can consider O being part of the first-neighbour shell for $\mathrm{Ca}$ when this distance is less than $2.65 \AA$. As shown in Table 2, the largest Ca-O bond distance within the first-neighbour shell is $<2.5 \AA$ when vaterite is optimised in the $\mathrm{P}_{5} 22$, Pbnm and $\mathrm{Ama} 2$ space groups, the second-neighbour shell being at $\mathrm{Ca}-\mathrm{O}>3 \AA$. In our new stable structures there are $\mathrm{Ca}-\mathrm{O}$ bonds that fall over a broader range, which is qualitatively more consistent with experimental EXAFS data for vaterite. ${ }^{1}$

15 The computed IR and Raman spectra of the lowest energy structure are in good agreement with the experimental findings with regard to frequencies, ${ }^{5,6,10,14}$ though a quantitative comparison of the peak intensities was not possible. Lattice modes and $\mathrm{CO}_{3}$ rigid rotations fall below $430 \mathrm{~cm}^{-1}$, in-plane and out-of-plane $\mathrm{CO}_{3}$ bending modes are found in the 640-740 and 830-840 $\mathrm{cm}^{-1}$ ranges, respectively. Symmetric and asymmetric C-O stretching modes lie in the 1060-1080 and 1380-1550 $\mathrm{cm}^{-1}$ ranges, respectively. However, no major difference was noticed with respect to the vibrational spectra computed for the other vaterite models. Hence this only confirms the 20 validity of the new structure, rather than providing a means of discriminating between candidates.

The vibrational thermodynamic functions, i.e. zero point energy, vibrational entropy and heat capacity contributions, were computed at $298 \mathrm{~K}$ for the minimum energy structures with the PBEsol functional. An 80-atom supercell was built for $P 2_{1} 2_{1} 2_{l}$-vaterite in order to allow for phonon dispersion, the other cells being large enough already for the purposes of this comparison. These results predict that $P 3_{2} 21$-vaterite is more stable than $P 6_{5^{-}}$,

${ }_{25} P 2_{1}{ }_{1}{ }_{1} 2_{1^{-}}$and $P 112_{1^{-}}$-vaterite by $1.0,2.6$ and $0.5 \mathrm{~kJ} / \mathrm{mol}$, respectively, in terms of free energy, whereas the corresponding enthalpy differences are $0.4,1.1$ and $0.6 \mathrm{~kJ} / \mathrm{mol}$ in favour of $P 3_{2} 21$-vaterite.

The variation in the relative stability of the various structures with the different functionals considered requires further comment. As already noted in previous works, ${ }^{23}$ the relative stabilities of phases having different densities are sensitive to the choice of functional due to systematic errors. In particular, the 
Table 4 Thermodynamics of vaterite relative to calcite (in $\mathrm{kJ} / \mathrm{mol}$ ) at $298.15 \mathrm{~K}$. Values are quoted for the PBEsol and PBEsol0 functionals based on either the most stable $P 3_{2} 21$ structure, or the Boltzmann-weighted average of all minima.

$\begin{array}{ccccc} & \Delta U & \Delta H^{298.15} & \Delta S^{298.15} & \Delta G^{298.15} \\ \text { PBEsol }\left(\text { P3 }_{2} 21\right) & 3.1 & 3.3 & 0.8 & 3.1 \\ \text { PBEsol (average) } & 3.4 & 3.6 & 0.4 & 3.5 \\ \text { PBEsol0 (P3221) } & 2.8 & 3.1 & 1.5 & 2.6 \\ \text { PBEsol0 (average) } & 3.2^{*} & & & \\ \text { Expt }^{31} & - & 3.6 & 1.3 & 3.2\end{array}$

*Relative populations from $\Delta G^{298.15}$ obtained with PBEsol

tendency of PBE, B3LYP and PBE0 to over-stabilise less dense structures has been documented. Given this, and the small magnitude of 5 the energy differences involved, it is important to calibrate the quality of the present results. To do this we have computed the thermodynamics of vaterite relative to calcite, as given in

Figure 1 Graphical representation of the hexagonal vaterite structures. A view of the $18 \mathrm{Ca}$ atoms in the unit cell, with the corresponding coordination polyhedra, projected on to the $a c$ plane. 6-, 7-, and 8-fold coordinated $\mathrm{Ca}$ atoms and polyhedra are shown in different shades of green; $\mathrm{C}$ and $\mathrm{O}$ atoms are grey and red, respectively.

For the case of the hexagonal $P 6_{5} 22$-structure the situation is more complicated as there are four different imaginary modes leading to distinct minima. As a result of the scans along these 10 four modes (all corresponding to symmetry-forbidden rotations of $\mathrm{CO}_{3}{ }^{2-}$ groups), three minimum energy structures, with space groups of $P 112_{1}, P 6_{5}$ and $P 3_{2} 21$, were obtained. A fourth structure (labelled in the following discussion as $s 4$ ) turned out to correspond to a $2^{\text {nd }}$-order saddle point and so was not further 15 analysed in detail.

$P 3_{2} 21$-vaterite, shown in Figure 1, is found to be the lowest energy structure (the unit cells and coordinates of all of the minima are reported in the Supplementary Information). Differences between $\mathrm{P}_{5} 22$-vaterite and the structures belonging 20 to its subgroups are quite small, mainly corresponding to more distorted positions of $\mathrm{O}$ atoms, whereas $\mathrm{Ca}$ and $\mathrm{C}$ atoms largely remain on the equivalent higher symmetry sites. This is shown by the varying coordination of $\mathrm{Ca}$ atoms and the bond length distribution.

${ }_{25}$ According to the $\mathrm{Ca}-\mathrm{O}$ distances in the other $\mathrm{CaCO}_{3}$ polymorphs, we can consider $\mathrm{O}$ being part of the first-neighbour shell for $\mathrm{Ca}$ when this distance is less than $2.65 \AA$. As shown in Table 2, the largest $\mathrm{Ca}-\mathrm{O}$ bond distance within the first-neighbour shell is $<$ $2.5 \AA$ when vaterite is optimised in the $\mathrm{P}_{5} 22, \mathrm{Pbnm}$ and $\mathrm{Ama} 2$ 30 space groups, the second-neighbour shell being at $\mathrm{Ca}-\mathrm{O}>3 \AA$. In our new stable structures there are $\mathrm{Ca}-\mathrm{O}$ bonds that fall over a broader range, which is qualitatively more consistent with experimental EXAFS data for vaterite. ${ }^{1}$

The computed IR and Raman spectra of the lowest energy 35 structure are in good agreement with the experimental findings with regard to frequencies, ${ }^{5,6,10,14}$ though a quantitative comparison of the peak intensities was not possible. Lattice modes and $\mathrm{CO}_{3}$ rigid rotations fall below $430 \mathrm{~cm}^{-1}$, in-plane and out-of-plane $\mathrm{CO}_{3}$ bending modes are found in the 640-740 and $40830-840 \mathrm{~cm}^{-1}$ ranges, respectively. Symmetric and asymmetric CO stretching modes lie in the $1060-1080$ and $1380-1550 \mathrm{~cm}^{-1}$ ranges, respectively. However, no major difference was noticed with respect to the vibrational spectra computed for the other vaterite models. Hence this only confirms the validity of the new 45 structure, rather than providing a means of discriminating between candidates.

The vibrational thermodynamic functions, i.e. zero point energy, vibrational entropy and heat capacity contributions, were computed at $298 \mathrm{~K}$ for the minimum energy structures with the
${ }_{50}$ PBEsol functional. An 80 -atom supercell was built for $P 2_{l} 2_{1} 2_{l^{-}}$ vaterite in order to allow for phonon dispersion, the other cells being large enough already for the purposes of this comparison. These results predict that $P 3_{2} 21$-vaterite is more stable than $P 6_{5^{-}}$, $P 2{ }_{1}{ }_{1} 2_{1^{-}}$and $P 112_{1^{-}}$-vaterite by $1.0,2.6$ and $0.5 \mathrm{~kJ} / \mathrm{mol}$, 55 respectively, in terms of free energy, whereas the corresponding enthalpy differences are $0.4,1.1$ and $0.6 \mathrm{~kJ} / \mathrm{mol}$ in favour of $P 3_{2} 21$-vaterite.

The variation in the relative stability of the various structures with the different functionals considered requires further 60 comment. As already noted in previous works, ${ }^{23}$ the relative stabilities of phases having different densities are sensitive to the choice of functional due to systematic errors. In particular, the

Table 4, based both on the lowest energy structure and for the Boltzmann-weighted average of the three distorted structures (i.e. 65 the three minima with $P 3_{2} 21, P 112_{1}$ and $P 6_{5}$ symmetries are averaged with weights of $0.40,0.33$ and 0.27 , respectively, computed based on their relative free energies). This demonstrates that this free energy difference is in good agreement with experiment for both PBEsol and PBEsol0. To put this in 70 perspective, the free energy difference for the previous ordered literature structures would have been in error by a factor of a third or greater. For further verification, the thermodynamics of aragonite relative to calcite computed at the PBEsol0 level gives $\Delta H^{298.15}, \Delta S^{298.15}$ and $\Delta G^{298.15}$ of $0.8(0.4), 3.5(4.3)$ and $-0.2(-0.8)$ $75 \mathrm{~kJ} / \mathrm{mol}$ (experimental values in parenthesis). Regardless of any systematic errors, $P 3_{2} 21$-vaterite turns out to be the most stable ordered structure with all the functionals considered. Furthermore, although the energy differences are small, the likely accuracy of the quantum mechanical methods is higher than for 80 many chemical reactions because of the close similarity of the states being compared.

\section{Conclusions}

In this work, the structure and the vibrational spectrum of both $\mathrm{P6}_{5} 22$ - and Pbnm-vaterite were computed at the DFT level (GGA 85 and hybrid functionals) by using all-electron quantum mechanical methods. Both of the previously proposed ordered structures for vaterite correspond to transition states, and therefore cannot be considered as representative minima on the potential energy surface. Five genuine minimum energy structures have been 90 obtained for vaterite, most of which have symmetries not 
previously examined for this phase. One of these configurations, with $P 3_{2} 21$ symmetry, is proposed as the best model for an ordered vaterite structure on the basis of energetic considerations. In reality, vaterite is known to exhibit structural disorder. This is 5 readily explained by the results of the present study, which demonstrates that there are at least three distinct minima whose energies and activation barriers for interconversion, given by the energy difference between the unstable $P 6_{5} 22$ transition state and the distorted minima, all are within the accessible range of 10 thermal energy at room temperature. Therefore the true structure of vaterite observed would almost certainly be a combination of three minima with symmetries of $P 112_{1}, P 6_{5}$ and $P 3_{2} 21$ that lead to a Boltzmann-weighted average structure close to the space group of $\mathrm{P}_{5} 22$. Our proposal that vaterite exchanges between 15 three distorted structures is consistent with the findings of Wehrmeister et al. that suggest an instanteously lower symmetry with three inequivalent carbonate environments. Conventionally, rotation of the carbonate groups about the $c$ axis of the hexagonal cell is assumed to be responsible for the disorder in vaterite. The 20 present results suggest that rotation of carbonate groups about axes that lie in the $a b$ plane also provides an alternative or additional mechanism for disorder. The final nature of the disorder may ultimately depend on the kinetics of the transformation from a precursor phase, as postulated for the ikaite 25 to vaterite conversion. ${ }^{32}$

\section{Acknowledgement}

The authors thank the Australian Research Council for funding through the Discovery grant DP0986999, as well as iVEC and NCI for providing computing resources.

\section{${ }_{30}$ Notes and references}

${ }^{a}$ Nanochemistry Research Institute, Department of Chemistry, Curtin University, GPO Box U1987, Perth, WA 6845, Australia; E-mail: raffaella@ivec.org

${ }^{b}$ Dipartimento di Chimica IFM, Università degli Studi di Torino and NIS 35 -Nanostructured Interfaces and Surfaces - Centre of Excellence, Via Giuria 7, 10125 Torino, Italy

$\dagger$ Electronic Supplementary Information (ESI) available: Fractional coordinates of the minimum energy vaterite structures as obtained with the PBEsol functional. See DOI: 10.1039/b000000x/

1 B. Hasse, H. Ehrenberg, J. C. Marxen, W. Becker and M. Epple, Chem. Euro. J., 2000, 6, 3679.

2 T. Ogino, T. Suzuki and K. Sawada, Geochim, et Cosmochim. Acta, 1987, 51, 2757.

3 J. D. Rodriguez-Blanco, S. Shaw and L. G. Benning, Nanoscale, 2011, 3, 265.

4 D. Gebauer, P. N. Gunawidjaja, J. Y. P. Ko, Z. Bacsik, B. Aziz, L. J. Liu, Y. F. Hu, L. Bergstrom, C. W. Tai, T. K. Sham, M. Eden and N. Hedin, Angewandte Chemie - International Edition, 2010, 49, 8889.

5 G. Behrens, L. T. Kuhn, R. Ubic and A. H. Heuer, Spectroscopy Lett., 1995, 28, 983.

6 C. Gabrielli, R. Jaouhari, S. Joiret and G. Maurin, J. Raman Spect., 2000, 31, 497.

7 H. J. Meyer, Zeitschrift für Kristallographie, 1969, 128, 183.

8 D. L. Bryce, E. B. Bultz and D. Aebi, J. Am. Chem. Soc., 2008, 130, 9282.

9 A. Le Bail, S. Ouhenia and D. Chateigner, Powder Diffraction, 2011, 26, 16

10 U. Wehrmeister, A. L. Soldati, D. E. Jacob, T. Häger and W. Hofmeister, J. Raman Spect., 2010, 41, 193
11 H. J. Meyer, Angewandte Chemie, 1959, 71, 678; H. J. Meyer, Fortschritte der Mineralogie, 1960, 38, 86.

12 J. D. C. McConnell, Mineralogical Magazine, 1960, 32, 535.

13 S. R. Kahmi, Acta Cryst., 1963, 16, 770.

14 M. Sato and S. Matsuda, Z. Krist., 1969, 129, 405.

15 W. F. Bradley, D. L. Graf and R. S. Roth, Acta Cryst., 1996, 20, 283.

16 L. Dupont, F. Portemer and M. Figlarz, J. Mater. Chem., 1997, 7, 797.

17 K. Medeiros, E. L. Albuquerque, F. F. Maia Jr, E. W. S. Caetano and V. N. Freire, Chem. Phys. Lett., 2007, 435, 59.

$18 \mathrm{~J}$. Wang and U. Becker, Am. Miner., 2009, 94, 380.

19 R. Dovesi, R. Orlando, B. Civalleri, C. Roetti, V. R. Saunders and C. M. Zicovich-Wilson, Zeitschrift für Kristallographie, 2005, 220, 571; R. Dovesi, V. R. Saunders, C. Roetti, R. Orlando, C. M. ZicovichWilson, F. Pascale, B. Civalleri, K. Doll, N. M. Harrison, I. J. Bush, P. D'Arco and M. Llunell, 2009, CRYSTAL09 User's Manual, University of Torino, Torino.

20 L. Valenzano, F. J. Torres, K. Doll, F. Pascale, C. M. Zicovich-Wilson and R. Dovesi, Zeitschrift für Physikalische Chemie, 2006, 220, 893.

21 J. P. Perdew, K. Burke and M. Ernzerhof, Phys. Rev. Lett., 1996, 77, 3865.

22 J. P. Perdew, A. Ruzsinsky, G. I. Csonka, O. A. Vydrov, G. E. Scuseria, L. A. Constantin, X. Zhou and K. Burke, Phys. Rev. Lett., 2008, 100, 136406.

23 R. Demichelis, B. Civalleri, P. D'Arco and R. Dovesi, Int. J. Quantum Chem., 2010, 110, 2260.

24 D. Spagnoli, K. Refson, K. Wright and J.D. Gale, Phys. Rev. B, 2010, $81,094106$.

25 C. Adamo and V. Barone, J. Chem. Phys., 1999, 110, 6158.

26 A. D. Becke, J. Chem. Phys., 1993, 98, 5648; P. Stephens, F. Devlin, C. Chabalowski and M. Frisch, J. Phys. Chem., 1994, 98, 11623.

27 C. G. Broyden, Journal of the Institute of Mathematics and its Applications, 1970, 6, 76; R. Fletcher, Computer Journal, 1970, 13, 317; D. Goldfarb, Mathematics of Computation, 1970, 24, 23; D. F. Shanno, Mathematics of Computation, 1970, 24, 647.

28 H. T. Stokes and D. M. Hatch, (2004). FINDSYM, stokes.byu.edu/findsym.html.

29 A. V. Radha, T. Z. Forbes, C. E. Killian, P. U. P. A. Gilbert and A. Navrotsky, Proc. Natl. Acad. Sci., USA, 2010, 107, 16438.

30 A. Bilic and J. D. Gale, Phys. Rev. B, 2009, 79, 174107

31 E. Königsberger, L. -C. Königsberger, H. Gamsjäger, Geochim. Cosmochim. Acta 1999, 63, 3105

32 C. C. Tang, S. P. Thompson, J. E. Parker, A. R. Lennie, F. Azough and K. Kato, J. Appl. Cryst., 2009, 42, 225. 